\title{
Die European Communication Conference (ECC) 2018 in Lugano
}

\author{
Fiona Fehlmann, Universität Basel, Seminar für Medienwissenschaft \& Fachhochschule \\ Graubünden, Institut für Multimediaproduction \\ fiona.fehlmann@unibas.ch
}

Note

Invited report for the Thematic Section on ECREA 2018 devoted to the $7^{\text {th }}$ European Communication Conference of the European Communication and Research Association (ECREA), held in Lugano from October $31^{\text {st }}$ to November $3^{\text {rd }}, 2018$.

Im vergangenen Jahr - vom 31. Oktober bis 4. November 2018 - hat in Lugano die 7.European Communication Conference (ECC) stattgefunden. Während vier Tagen präsentierten 983 internationale ReferentInnen laufende oder abgeschlossene Forschungsprojekte zum Themenkomplex: Centres and Peripheries: Communication, Research, Translation. Mit 1248 Teilnehmenden war diese Tagung in Lugano die bisher grösste ihrer Art.

Im Allgemeinen zeigte sich, dass insbesondere in vier Forschungsbereichen bzw. Sektionen Panels stattgefunden haben: Erstens im Bereich Journalism Studies (29 Panels), zweitens im Bereich Communication and Democracy (21 Panels), drittens im Bereich Digital Culture and Communication (21 Panels) und viertens im Bereich Political Communication (21 Panels). Mit zahlreichen zusätzlichen Panels über diese Forschungsbereiche hinaus waren auf jeden Fall für alle Teilnehmenden viele interessante Einblicke in Forschungsprojekte möglich. Hinzu kamen während den Pausen spannende, vertiefende Diskussionen mit Kolleginnen und Kollegen in den Gängen und Hallen des Palazzo dei Congressi. Eine nicht ganz unbedeutende Nebensache durfte man sich in diesen Pausen ebenfalls nicht entgehen lassen: Einen richtig guten caffe - 7000 wurden in den vier Tagen ausgeschenkt.

Neben den ReferentenInnen für die jeweils parallel stattfindenden 214 Panels, lud die Konferenz ausserdem drei herausragende Keynote-Rednerinnen nach Lugano ein.
Eszter Hargittai eröffnete die Tagung mit ihrer Präsentation The online participation divide. Hargittai untersucht in ihrer Forschung, welche Faktoren (z.B. internet skills, Geschlecht oder sozioökonomischer Status) die Qualität und Effektivität der Onlinepartizipation beeinflussen.

Die zweite Rednerin am ersten Konferenztag war Lina Dencik mit ihrer Präsentation Resistance in the datafied society: From data ethics to data justice. Ihre Forschung bewegt sich im Spannungsfeld zwischen digitaler Überwachungssysteme, Datenschutzpolitik und Privatsphäre.

José van Dijck hielt schliesslich am Freitag ihre Keynote zum Thema: The geopolitics of platforms: Lessons from $\mathrm{Eu}$ rope. In ihrer Präsentation diskutierte sie Implikationen, welche grossen Plattformanbieter - die mehrheitlich aus dem amerikanischen oder chinesischen Kontext stammen - beispielsweise auf die europäische öffentliche Sphäre oder auf das europäische Gesundheits- und Transportsystem haben.

Mit der Teilnahme an der European Communication Conference in Lugano konnte man viele interessante Einblicke in die internationale Kommunikationsforschung gewinnen und nicht zuletzt auch Bezugs- und Anschlusspunkte für die eigene Forschung entdecken. Meine Teilnahme als eine von 350 Doktorierenden war somit ein voller Erfolg! Nochmals ein grosses grazie mille an das lokale Organisationsteam der Università della Svizzera Italiana (USI) und ich schaue schon freu- 
dig auf die nächste European Communication Conference 2020 in Braga, Portugal!

Quelle: ECC 2018 Lugano: The final report. Abgerufen unter: https://ecrea.eu/ Newsletter/6947145 (19.12.2018).

\section{L'European Communication Conference (ECC) 2018 de Lugano}

La $7^{\mathrm{e}}$ European Communication Conference (ECC) de l'European Communication Research and Education Association (ecrea) s'est tenue du 31 octobre au 4 novembre 2018 à Lugano. Durant quatre jours, 983 intervenant·e.s ont présenté leurs recherches autour du thème: Centers and Peripheries: Communication, Research, Translation. Réunissant 1248 participant.e.s, cette conférence a été la plus importante du domaine jusqu'à ce jour.

Couverts par 21 panels chacun, les domaines de recherche les plus représentés ont été Journalism Studies; Communication and Democracy; Digital Culture and Communication; et Political Communication. De nombreux autres panels portaient sur d'autres domaines de recherche. Pendant les pauses, qui ont eu lieu dans les couloirs et les halls du Palazzo dei Congres$s i$, les conversations animées entre les participant.e.s ont été incontournables. L'excellent caffè a sans doute joué un rôle non négligeable: plus de 7000 personnes ont été servies en quatre jours.

En plus des 214 panels parallèles, le programme de Lugano comportait trois conférences plénières:

Eszter Hargittai, de l'Université de Zurich, a ouvert le congrès avec une présen- tation intitulée "The online participation divide". S'appuyant sur ses recherches, elle a mis en lumière les facteurs influençant la qualité et l'efficacité de la participation en ligne, tels que les compétences médiatiques, le genre ou le statut socioéconomique des individus.

Lina Dencik est également intervenue en plénière le premier jour. Sa conférence, intitulée "Resistance in the datafied society: From data ethics to data justice", a souligné les tensions entre les systèmes de surveillance numérique, les politiques de protection des données et la sphère privée.

Enfin, José van Dijck a donné vendredi une conférence sur "The geopolitics of platforms: Lessons from Europe". Elle a souligné l'impact des grands opérateurs de plateformes - dont la plupart sont issus du contexte américain ou chinois - sur la sphère publique européenne, ainsi que sur les systèmes de santé et de transport en Europe.

En tant que l'un des 350 doctorant.e.s présent.e.s à l'European Communication Conference, j'ai bénéficié de précieux éclairages sur l'état des recherches internationales en communication, ainsi que de nombreuses références et contacts utiles à mon travail. C'est la raison pour laquelle je me réjouis d'ores et déjà de la prochaine European Communication Conference, qui aura lieu à Braga au Portugal en 2020! Grazie mille à toute l'équipe d'organisation de l'Università della Svizzera Italiana (USI).

Le rapport officiel de l'ecrea se trouve sur: https:// ecrea.eu/Newsletter/6947145 (19.12.2018). 